\title{
Numerical simulation of the betatron magnetic field using ELCUT software
}

\author{
Ivan Zatonov ${ }^{1, \mathrm{a}}$ and Mikhail Shtein ${ }^{1}$ \\ ${ }^{1}$ National Research Tomsk Polytechnic University, 634050 Tomsk, Russia
}

\begin{abstract}
The article examines the possibility of using software kits to solve multi-physical tasks in betatron engineering. It emphasizes radiation control in aerospace industry using smallsize pulse betatron as a source of ionizing radiation. Main characteristics of the betatron magnetic field are calculated using Elcut software kit. Dependence of calculation accuracy on the grid quantity is studied. Focusing properties of the betatron magnetic field are analyzed.
\end{abstract}

\section{Introduction}

Aerospace industry plays a significant role in ensuring military security, scientific and economic development of the Russian Federation. At this stage of development, Russia moves from sustaining the previous level of aerospace industry to new ambitious projects aiming at a higher production potential of this area [1]. Such an important role in the Russian strategy of military and technical and economic development demands a high level of reliability from the aerospace industry, which depends, first of all, on using instruments and methods of non-destructive testing at all production stages.

Owing to its increased feedback, radiation control is widely used for inspection of aerospace industry related products. The point of the digital X-ray method is the imaging of the object structure identifying foreign material and its geometry in the structure of the control object. In the last few years, similar techniques have been used to solve tasks of the aviation security. Inspection of largesize articles and air containers requires high energy X-ray sources now-a-days represented by betatrons and linear accelerators [2]. The distinctive feature of betatrons is small size and low cost, which allows them competing on the market regardless of the low dose rate.

The first Russian betatron for the energy of $4 \mathrm{MeV}$ was launched in Tomsk Polytechnic University in 1948. Since then, TPU is the reference University for betatron engineering not only in Russia but worldwide.

\footnotetext{
${ }^{a}$ Corresponding author : ivan.zatonov@yandex.ru
} 


\section{Experimental procedure}

\subsection{Betatron setting}

During betatron manufacturing, there are usually difficulties related to its adjustment, i.e. finding magnetic field parameters to achieve the rated electron energy and maximum dose rate.

There are several ways to adjust betatrons:

- Adjustment by searching parts that contribute into distribution of the betatron magnetic field and measurement of the dose rate for each variant. To ensure correct adjustment and maximum possible dose rate, dozens or hundreds of combinations are required sometimes.

- Magnetic measurements and change of magnetic field parameters in inter-polar gap based on the results of measurements. This brings a good result in a few combinations.

The principle of betatron operation is based on the fact that an electron can move along a circular orbit of the constant radius gaining energy in the magnetic field that decreases in radius and increases in time. Energy increment over one turn is proportional to the speed of magnetic flux change inside the orbit. Any deviation of the electron from the constant orbit results in bringing it back to this orbit by magnetic forces. That is why its radius $r_{0}$ is called equilibrium.

One of the main tasks of adjustment is to find the optimum radius of the equilibrium electron orbit as this radius determines both accelerated electron energy, and area of magnetic focusing forces, hence their quantity.

It is possible to find the equilibrium radius using a simple condition called "two to one relation" in the betatron theory [3].

$$
2 B_{z}(r)=\bar{B}
$$

where $B_{z}(r)$ is the induction of the magnetic field on the equilibrium radius $r, \bar{B}$ is the average induction in the circle $r_{0}$.

The same values that are found through magnetic measurements determine the energy of accelerated electrons

$$
E=\sqrt{\left(300 r_{0} B_{z}\left(r_{0}\right)\right)^{2}+\mathrm{E}_{0}^{2}}-\mathrm{E}_{0}
$$

where $\mathrm{E}_{0}$ - is the electron rest energy, which is $0,511 \mathrm{MeV}$.

The results of magnetic measurements are used to determine the radius dependence of the magnetic field decay index [4].

$$
n=-\frac{\mathrm{d}\left(B_{z}(r)\right) r}{B_{z}(r) \mathrm{d} r} .
$$

The potential function of magnetic field tells better about its focusing properties [5]

$$
V_{\mu}=\frac{\mathrm{e} A^{2}}{2 \mathrm{mc}^{2}}=\frac{\mathrm{e}}{2 \mathrm{mc}^{2}}\left(\frac{r \bar{B}}{2}\right)^{2}
$$

where $\mathrm{e}$ - is the electron charge, $A$ - is the vector magnetic potential, $\mathrm{m}$-is the electron mass, $\mathrm{c}-$ is the speed of light. 


\subsection{Numerical simulation}

The task of this work was to demonstrate the possibility to achieve the magnetic field characteristics for actual betatron designs with the required accuracy using numerical simulation as compared to time-consuming magnetic measurements. Numerical simulation allows significantly reduce the time of betatron adjustment.

Such a simulation can be done using software kits designed for finite element analysis in different areas of physics and engineering, including multi-physical tasks. The currently used kits are Ansys, Comsol Multiphysics, Elcut and so on.

Software kit Elcut was used for numerical simulation of the main characteristics of the betatron magnetic field as being most simple and easily understood. The design of a small-size MIB-10 betatron was used for analysis.

The geometry of the betatron magnet is shown in Figure 1.

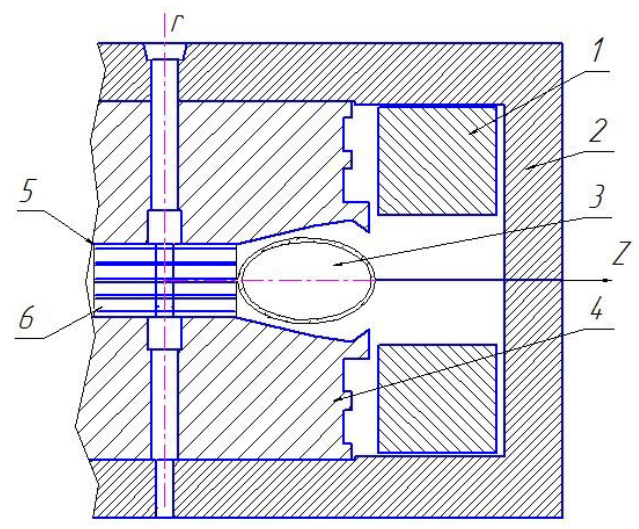

Figure 1. Geometry of a betatron, symmetric to $r$ axis, where 1- coil with 7500 ampere-turns current; 2- reverse magnetic conductor; 3- betatron X-ray chamber; 4- magnetic pole pieces; 5 - five identical magnetic spacers; each $2.4 \mathrm{~mm}$ thick; 6 - four magnetic pies; each $8 \mathrm{~mm}$ thick.

With account for the axially symmetric design of the MIB-10 betatron, a geometric model was made in Elcut software kit followed by setting the physical parameters of objects. The work with geometric model is done in a cylindrical coordinate system in half-plane $\mathrm{zr}(r \geq 0)$ (Figure 2).

Since the finite-element method is used to find the solution in the software, the grid application is a mandatory stage of modelling. The smaller the grid space, the higher the accuracy of calculations but the longer the time. Initially a number of calculations was done, the spaces being $2,1,0.5$ and 0.25 $\mathrm{mm}$. The results do not change beginning from $1 \mathrm{~mm}$ space. Setting an option of automatic selection of the grid space with 13403 grid points had the same result. The grid at automatic selection is shown in Figure $2 b$.

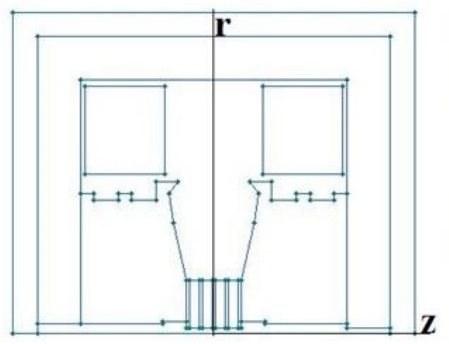

a.

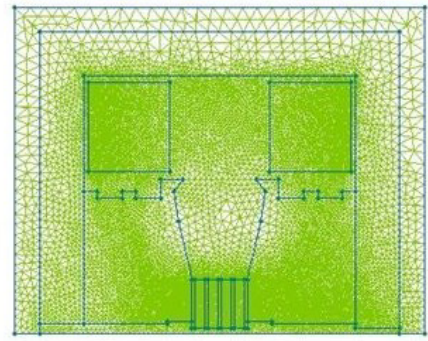

b.

Figure 2. a. Geometric model of a betatron in axially symmetric class of the model; b. Automatic sampling of the grid applied to the studied betatron model. 


\section{MATEC Web of Conferences}

The Elcut software solves the Poincare equation for vector magnetic potential A but in the axially symmetric task it calculates the related flux function $\Phi=r A$. Then vector components of the magnetic induction are found by numerical differentiation $B_{z}$ and $B_{r}$.

First, the value of the radius of equilibrium orbit was determined based on assumption that the orbit is completely within the $\mathrm{z}$ plane. For this, the contour at $\mathrm{z}=0 \mathrm{~mm}$ on sector $r \in[43 ; 130] \mathrm{mm}$ was studied by the software, and induction values depending on radius $B_{z}(r)$ were found.

The average induction $B_{c p}$ in not calculated by the software; it was found as follows:

$$
\bar{B}(r)=\frac{\Phi(r)}{\mathrm{S}}=\frac{\int \mathrm{d} \Phi}{\mathrm{S}}=2 \frac{\int_{0}^{r} B_{z}(r) \pi r \mathrm{~d} r}{\pi r^{2}},
$$

where $\Phi(r)$ - is the magnetic flux of radius $\mathrm{r}, \mathrm{S}-$ is the area of this circle.

Since data are presented as a table, the Simpson formula was used for calculation of the certain integral:

$$
\bar{B}\left(r_{i}\right)=\frac{\sum_{i=1}^{\mathrm{n}} \pi\left(r_{i+1}^{2}-r_{i}^{2}\right) \frac{B_{z}\left(r_{i+1}\right)+B_{z}\left(r_{i}\right)}{2}}{\pi r_{i}^{2}} .
$$

The calculated values of the average induction $\bar{B}$ correspond to the average radius:

$$
\bar{r}_{i}=\frac{r_{i}+r_{i+1}}{2} .
$$

\section{Results}

The cross point of the chart of induction distribution $B_{z}<\frac{\bar{B}}{2}$ is the value of the equilibrium radius (Figure 3). With the above-mentioned sizes of the pie stack, the value of the equilibrium radius is $101,8 \mathrm{~mm}$, the induction on it being $0,278 \mathrm{Tl}$.

The value of the electron energy in the given circuit was determined in parallel to the equilibrium radius. Formula (2) was used for calculation and $8 \mathrm{MeV}$ value was obtained, which was close to the expected value.

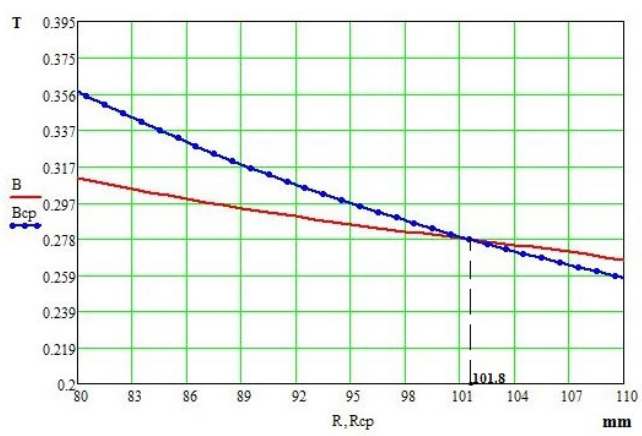

Figure 3. Determining the equilibrium radius. 
The indicator of magnetic field strength decay in $z=0$ plane calculated by formula (3) is given in figure 4 .

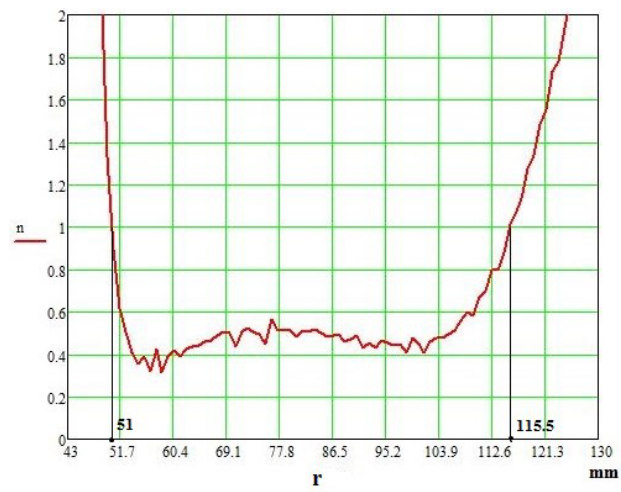

Figure 4. Radius $r$ dependence of the decay indicator $n$.

Presence of focusing forces in radial and axial directions at the same time is guaranteed when $0<n<1$ inequality is satisfied. According to the chart, the stable orbits are those with radii from 51 to $115,5 \mathrm{~mm}$.

As already said, the potential function $V_{\mu}$ is a visual and complete characteristic that describes focusing properties of the magnetic field. The surface described by the potential function is normally called a potential hole [6].

The potential hole made for $\mathrm{z}=0$ plane is shown in Figure 5.

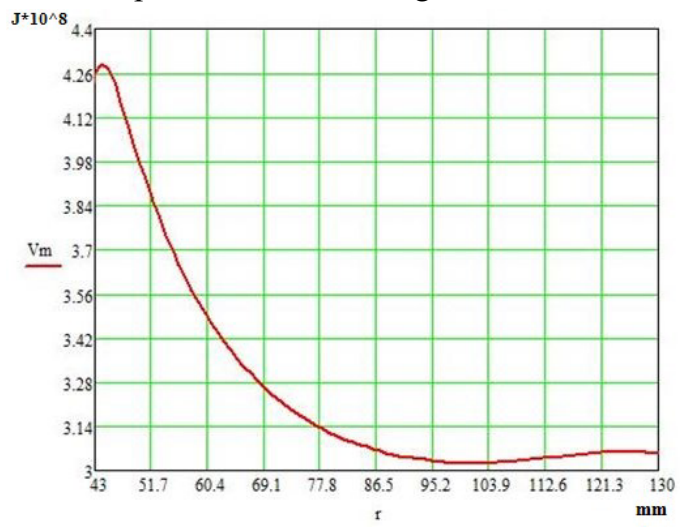

Figure 5. Potential hole for $\mathrm{z}=0$ plane.

As it appears from the betatron theory, the minimum of the potential hole is on the equilibrium radius.

To have a comprehensive idea of focusing forces along the whole cross-section of inter-gap space including along the cross-section of the accelerating chamber, the potential hole is calculated as a function of two coordinates, and usually presented as lines of the same level on the plane. The outline in Figure 6 shows an area, where values of the potential function were calculated, resulting in the potential hole. This picture also demonstrates distribution of the magnetic field lines. 


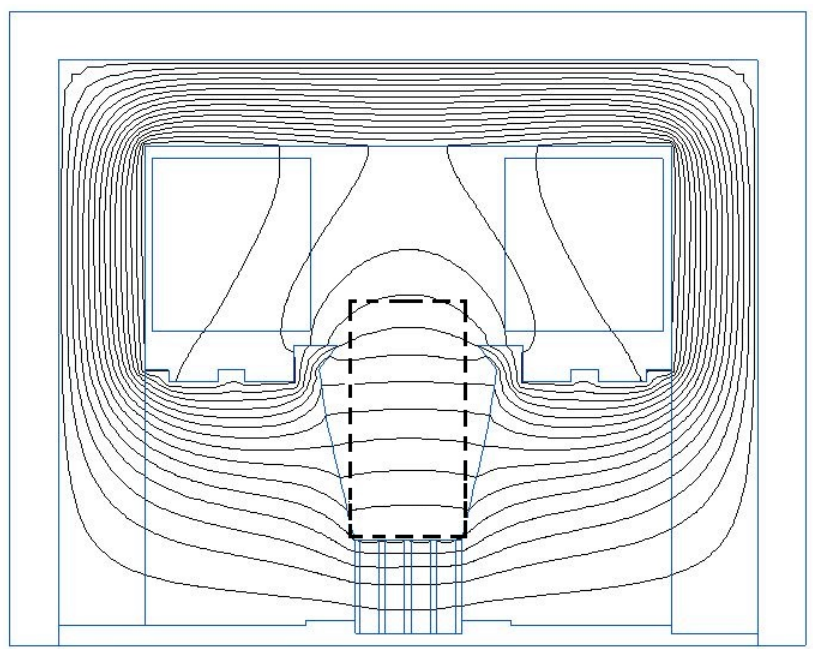

Figure 6. Area of the model used for calculation of potential function.

For the specified conditions of calculations, we get a picture of the potential hole presented in Fig. 7. The red outline shows a vacuum chamber that in theory can have any electron orbits inside of it. The green contour indicates the chamber injector. The latter reduces the effective chamber volume but a betatron cannot work without it.

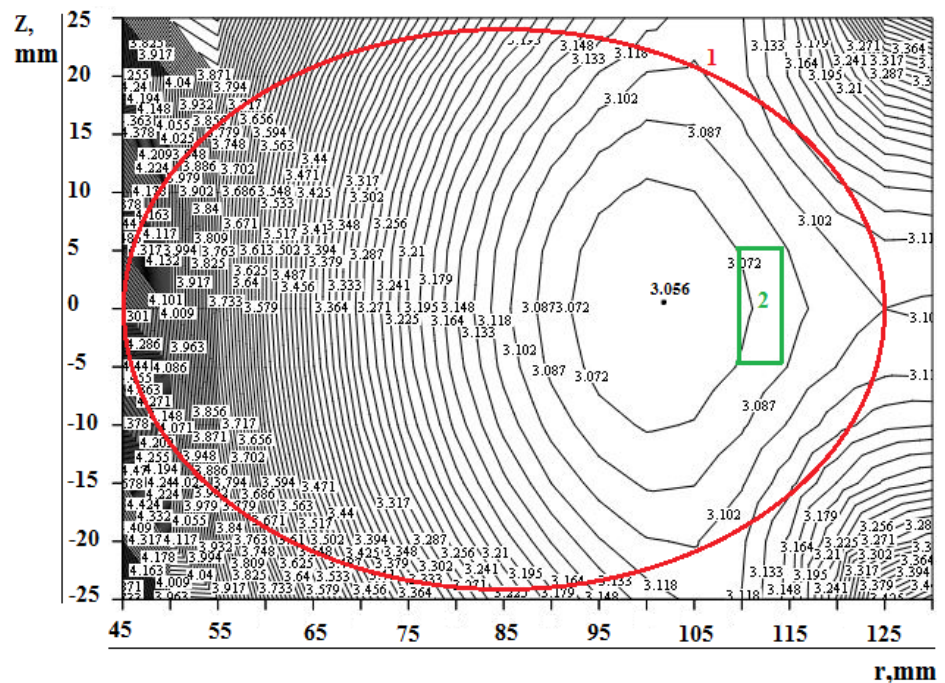

Figure 7. Chart of potential function lines: 1 - inscribed X-ray chamber; 2 - injector.

\section{Conclusion}

Elcut software suite intended for solving multiphysical tasks by the finite-element method is an effective instrument for numerical modelling of the betatron magnetic field. In many cases, it can replace the process of magnetic measurements and reduce the time of betatron adjustment. Computer time spent for the modelling was not long and took 30 seconds. Processing of the results takes much longer. In this respect, the problem number one is to import the result data of Elcut into more powerful modern programs of data store processing, and create a command file for complete automatization of the process of getting the results in a convenient and easy-to-use form. 
As for specific results of the presented magnet modelling, it is obvious that the size of the pie stack was incorrectly selected. The equilibrium radius is close to the injector and only a small part of the chamber cross-section is effective. The radius is set off outwards and the first line of the equal level is in contact with the injector (Fig. 7). Only within this line stable electron orbits may exist. Electrons whose oscillating energy is higher than potential energy of the first level, can make several turns but ultimately they will overcome this barrier and hit the injector.

In order to reduce the equilibrium radius, it is necessary to decrease the height of the pies and increase thickness of non-magnetic spacers leaving the gap between the pole pieces unchanged. By doing several numerical simulations, one can determine the acceptable position of the equilibrium radius and hence the structure of the pie stack.

\section{References}

1. A. S. Karpov, Natsionalnyie interesyi: prioritetyi i bezopasnost 12, 43 (2008)

2. J. Accardo, M. Ahmad Chaudhry, Journal of Radiations Research and Applied Sciences 7, 198 (2014)

3. K. S. Grishin, Izvestiya TPI 87, 95 (1957)

4. Timothy W. Koeth, Physics Procedia 66, 622 (2014)

5. B. N. Rodimov, Izvestiya TPI 87, 3 (1957)

6. D. A. Noskov, Izvestiya TPI 87, 151 (1957) 